\title{
Terra mission operations: Launch to the present (and beyond)
}

\author{
Angelita Kelly*a, Eric Moyer ${ }^{\mathrm{a}}$, Dimitrios Mantziaras ${ }^{\mathrm{a}}$, Warren Case $^{\mathrm{b}}$ \\ ${ }^{a}$ NASA-GSFC, 8800 Greenbelt Rd., Greenbelt, MD, USA 20771 \\ bColumbus Technologies, Inc., 7500 Greenway Center Dr., Greenbelt, MD, USA 20771
}

\begin{abstract}
The Terra satellite, flagship of NASA's long-term Earth Observing System (EOS) Program, continues to provide useful earth science observations well past its 5-year design lifetime. This paper describes the evolution of Terra operations, including challenges and successes and the steps taken to preserve science requirements and prolong spacecraft life. Working cooperatively with the Terra science and instrument teams, including NASA's international partners, the mission operations team has successfully kept the Terra operating continuously, resolving challenges and adjusting operations as needed. Terra retains all of its observing capabilities (except Short Wave Infrared) despite its age. The paper also describes concepts for future operations.

This paper will review the Terra spacecraft mission successes and unique spacecraft component designs that provided significant benefits extending mission life and science. In addition, it discusses special activities as well as anomalies and corresponding recovery efforts. Lastly, it discusses future plans for continued operations.
\end{abstract}

Keywords: Terra, EOS, AM1, Spacecraft Operations, Flight Operations, Mission Operations, safety, instruments, cooperation

\section{INTRODUCTION}

Terra is a joint project between the United States, Japan, and Canada. It was launched on December 18 , 1999 from Vandenberg Air Force Base aboard an International Launch Services Atlas booster into a Sun-synchronous orbit around the Earth [1]. It joined the Landsat-7 satellite (launched in April 1999) and the Landsat-5 satellite (launched in March 1984), both of which are operated by the United States Geological Survey (USGS). A year later, Argentina's SAC-C satellite and NASA's technology demonstration satellite, EO-1, were launched into the same orbit, thus forming the Earth observing Morning Constellation.

The Terra mission marked the beginning of the comprehensive study of clouds, water vapor, small particles in the atmosphere (i.e., aerosols), trace gases, land surface and oceanic properties, as well as the interaction between them and their effects on the Earth's energy budget and climate.

After reaching its proper orbit at 10:30 \pm 15 minutes Mean Local Time (MLT), Terra began the collection of continuous, long-term, calibrated measurements of global processes on February 24, 2000. Terra and subsequent EOS missions (Aqua, Aura, and ICESat) form the core of NASA's Earth Science Program. The goal of the program is to turn NASA's spacebased observing technology and scientific expertise to the study of the planet Earth as an integrated system of land, ocean, atmosphere, ice, and biological processes. By viewing the Earth from space, scientists can begin to understand how the systems work and how they interact.

\subsection{Spacecraft}

The Terra spacecraft (Fig. 1) was built at the Lockheed Martin Missiles \& Space facility in Valley Forge, PA for NASA Goddard Space Flight Center, MD. The U.S. provided the spacecraft and three instruments developed by NASA Field Centers. It also includes sensors from NASA's International Partners, Japan and Canada, demonstrating cooperation for Terra's international scientific research.

*angelita.c.kelly@nasa.gov; phone 1301 614-5317; www.nasa.gov 


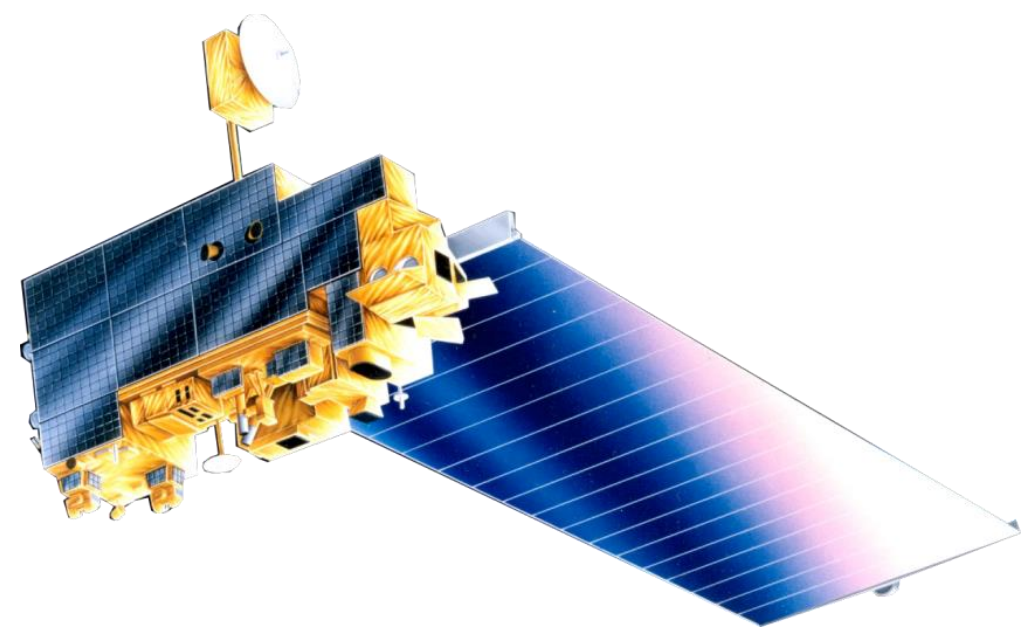

Figure 1. Terra Satellite (Artist's concept)

\subsection{Instruments}

Terra's instruments comprise of eight separate sensors with a range of resolutions that gather data on clouds, aerosols, the Earth's radiative balance, and the Earth's surface properties and interaction with the atmosphere:

- ASTER, the Advanced Spaceborne Thermal Emission and Reflection Radiometer, provides high-resolution images of the land surface, water, ice, and clouds using three separate sensor subsystems (Shortwave Infrared (SWIR); Thermal Infrared (TIR); Visible and Near Infrared (VNIR) covering 14 multi-spectral bands from visible to the thermal infrared. Terra was provided by Japan's Ministry of Economy, Trade, and Industry. The SWIR cooler anomaly in 2008 resulted in permanent loss of further usable data

- CERES, the Clouds and Earth's Radiant Energy System, measures Earth's radiation budget and atmospheric radiation from the top of the atmosphere using a broadband scanning radiometer with bolometers detectors. CERES was built at NASA's Langley Research Center. It has 2 instruments: CERES-Aft and CERES-Fore.

- MISR, the Multi-angle Imaging Spectro-Radiometer, measures top-of-atmosphere cloud and surface angular reflectance functions, surface bidirectional reflectance distribution function (BRDF), aerosol, and vegetation properties using four spectral bands in each of nine imaging cameras oriented at different angles. MISR was developed at NASA's Jet Propulsion Laboratory.

- MODIS, the Moderate Resolution Imaging Spectro-Radiometer, measures biological and physical processes on land and the ocean using a cross-track scanning multi-spectral radiometer with 36 spectral bands from visible to the thermal infrared. MODIS was developed at NASA's Goddard Space Flight Center.

- MOPITT, the Measurements of Pollution in the Troposphere, measures carbon monoxide and methane in the troposphere using correlation spectroscopy with pressure modulated and length modulated gas cells. MOPITT was built in Canada and is a joint cooperation between the Canadian Space Agency, the University of Toronto, and the National Center for Atmospheric Research (NCAR) in Boulder, Colorado.

\section{SPACECRAFT SUBSYSTEM PERFORMANCE}

Terra's subsystems have performed very well since launch. After almost 15 years on orbit, Terra continues to operate on primary hardware.

\subsection{Propulsion Subsystem}

The Propulsion Subsystem is performing nominally. A total of 122 propulsive maneuvers have been executed since the initial series of post-launch activities to insert Terra into the proper mission orbit. The types of maneuvers include Drag 
Make-up (DMU) maneuvers performed as needed to compensate for the effects of atmospheric drag and Inclination Adjust Maneuvers (IAMs) performed twice per year to maintain the proper sun-synchronous precession rate.

Based on projected nominal DMU maneuvers and IAMs, Terra has sufficient propellant to continue nominal operations at the $705 \mathrm{~km}$ orbit height through August 2019 (Fig. 2).

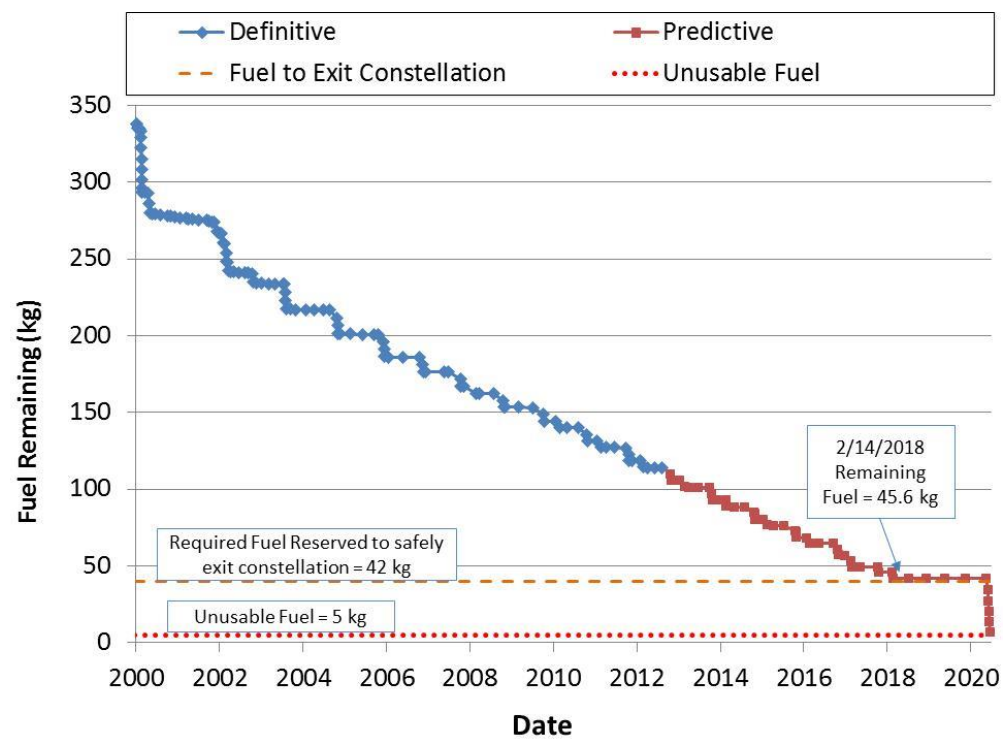

Figure 2. Terra Predicted Propellant Usage

\subsection{Electrical Power (EPS) Subsystem}

The Terra battery is divided into two assemblies, the Hex Bay Battery (BBAT) and Power Equipment Module Battery (PBAT) with 54 nickel-hydrogen $(\mathrm{NiH} 2)$ cells per each battery (for a total of 108 cells). Each battery is managed separately. The batteries have behaved nominally, other than 2 anomalies that both occurred on October 13, 2009. These 2 failures (a single battery cell failure anomaly and a battery heater control anomaly, both in the BBAT) were likely caused by Micrometeoroid or Orbital Debris (MMOD), based on an observed attitude disturbance and failure analysis. If required, each battery by itself has sufficient capacity to continue the mission, although there would be some impacts to operations.

Battery pressure, temperature and voltage trends are all within an acceptable range, and there are currently no known issues (other than the two aforementioned anomalies), that are expected to affect the life of the batteries. The solar array power output has far exceeded the maximum total power required since launch, including for all maneuvers, anomalies, and special events. The solar array continues to provide excess power even though it lost $1 / 24^{\text {th }}$ of its capability in 2000 due to a failed shunt circuit and a -10 degree offset. No other issues affecting the Solar Array or the Solar Array Drive mechanism have been observed.

\subsection{Guidance, Navigation \& Control (GN\&C) Subsystem}

There are no issues with the Inertial Reference Units (IRU), Reaction Wheel Assemblies (RWA), or any other components of the Attitude Determination and Control Subsystem (ADAC). The average IRU gyro motor current consistently stays well below the yellow limit for all 3 IRUs. The average estimated wheel speed friction for the 4 RWAs has remained well below the yellow limit, and has not reached levels that would cause concern even during safehold events or special operations like the Deep Space Calibration Maneuvers. The Fault Detection Isolation and Recovery has been performing well.

\subsection{Command \& Data Handling (C\&DH) Subsystem}

The Terra Solid State Recorder (SSR) provides on-board data recording and storage with a capacity of 172.5 Gbits. Most of the storage capacity resides within two Data Memory Units (DMU) supporting separate buffers for the five Terra 
instruments and a Trash Buffer for error-logging and diagnostic data. Additionally, a Data Control Unit (DCU) houses an engineering or "Housekeeping" buffer for recording and storage of data pertaining to operating parameters and component health and safety. Since launch, Terra has experienced multiple incidents in which one or more Printed Wire Assembly (PWA) boards (which make up the DMUs) become incapable of recording and storing data. These incidents are caused by the PWA over-current protection circuitry, which turns off individual PWAs as a result of Single Event Upsets (SEU) or other effects. The current reduction in SSR storage capacity is approximately $26.3 \mathrm{Gbits}$, or $15.5 \%$ of the maximum science data storage capacity. It is predicted that there will be a loss of an additional 4 PWAs by 2016. Demonstrating cooperation, the ASTER team agreed to reduce their maximum science data collection every two orbits by 4.2 minutes in order to minimize the impacts to MODIS and MISR science data capture and operations..

\section{SPACECRAFT-UNIQUE SUBSYSTEM COMPONENT DESIGN}

\subsection{MODIS Direct Broadcast (Build it and they will come)}

The Terra Direct Access System (DAS) provides the capability to downlink realtime data via X-Band. This Direct Broadcast (DB) capability was in response to the need to provide timely data (within 3 hours of onboard collection) for weather forecasting. Terra's MODIS DB data has proven to be extremely popular and beneficial for operational use throughout the world for weather forecasting, fire management and monitoring, disaster management and monitoring, fishery, military application, etc. Today, there are over 200 identified ground stations that receive and process Terra DB data. The processed data are distributed further to various government and private institutions and users.

\subsection{Solar Array (SA) blanket}

Terra has a large lightweight single solar array (Fig. 3) that is 29 feet long by 16 feet wide ( $9 \mathrm{~m}$ by $5 \mathrm{~m}$ ). It used a new design which reduced mass to approximately $1 / 3$ when compared with other fixed solar array wing arrays of similar size. The gallium arsenide on germanium $(\mathrm{GaAs} / \mathrm{Ge})$ solar cells attached to the blanket provide sufficient power to exceed the $7.5 \mathrm{~kW}$ Beginning of Life requirement and its performance has far exceeded the $5.0 \mathrm{~kW}$ End of Life requirement at 5 years.

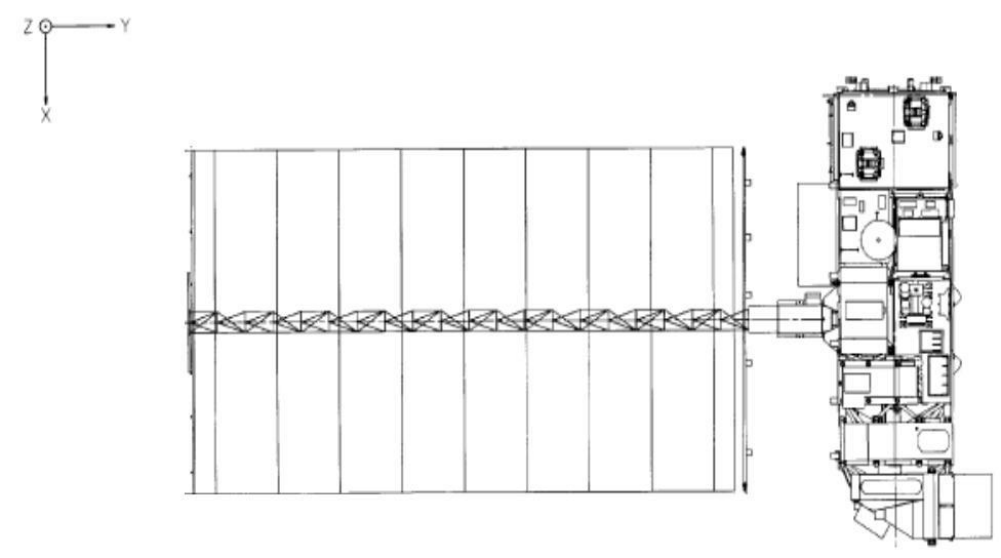

Figure 3. Terra Spacecraft with Solar Array (SA) Deployed (Mast shown)

The reduction in weight of the single solar array wing allows for alignment of translation thrusters through the center of mass minimizing the complexity of yawing the spacecraft to perform nominal drag make up maneuvers. The overall design concept was demonstrated on Space Shuttle flight STS 41-D in 1984 [2 and 3].

\subsection{Regulated 120V Bus}

The Terra spacecraft was the first orbiting 120V-regulated bus implemented by NASA (Fig. 4). This was done since the original Terra (AM-1) and its 20 Earth Observing System (EOS) instruments were considered to fly as an attached platform 
on Space Station Freedom (precursor to the International Space Station), so the EOS program worked closely with the Space Station Freedom sharing component designs. The 2 programs were eventually decoupled, but several of the shared component designs were fortunately already incorporated into the Terra spacecraft design.

The $120 \mathrm{~V}$ regulated bus is used to handle the high power demands for the spacecraft bus and instruments, reduce the spacecraft mass, reduce parasitic losses, and reduce bus noise [4].

The Power Distribution Unit (PDU) regulates the spacecraft bus power by providing control signals to the Solar Array (SA) Sequential Shunt Unit (SSU) and the Battery Power Conditioner (BPC). The PDU supports the spacecraft bus power demands by utilizing available SA power first; if the load is greater than what the SA can provide, then the PDU sends signals to both BPCs to pull the needed power from each battery to maintain the spacecraft bus at $120 \mathrm{~V}$. If the SA has excess power, the PDU will signal SSU to provide power to charge batteries based on each battery charge management settings. If the SA still has excess power, the SSU will shunt the power back to the array.

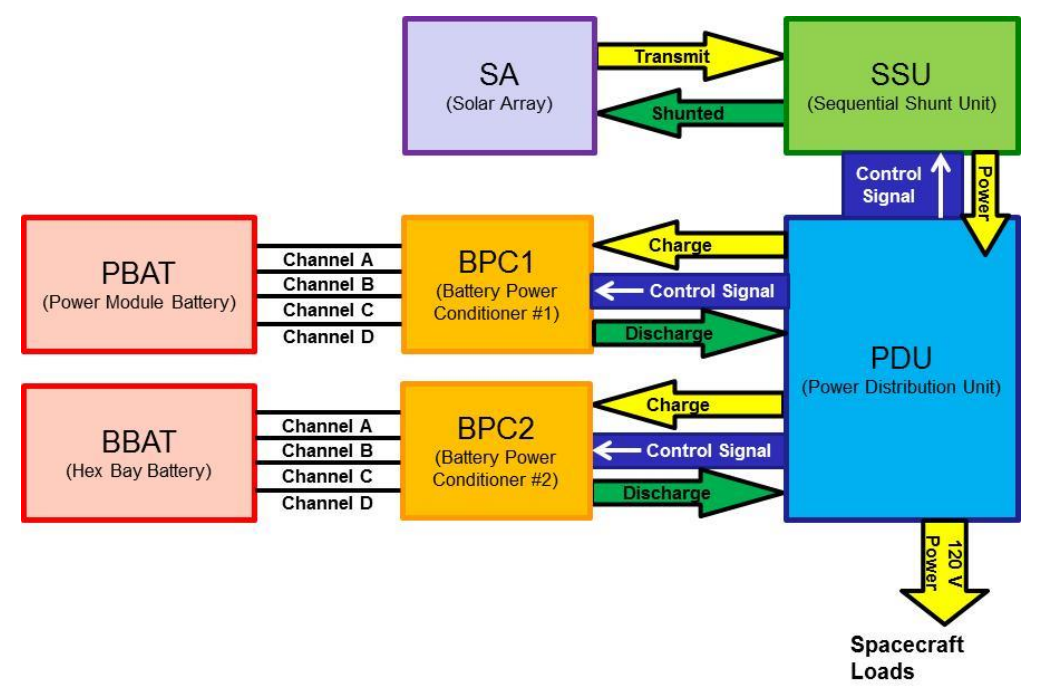

Figure 4. Terra Regulated 120 V Bus Block Diagram

\subsection{Battery Power Conditioner (BPC)}

There are 2 BPCs with 4 channels each. Each BPC is dedicated to one battery. The channels flow current to the battery. The BPC performs both charge and discharge functions. Charging of each battery is managed separately within the PDU. The PDU sends signals to each BPC based on each selected battery charge rate and voltage/temperature curve. Discharge is shared across all enabled BPC channels.

\subsection{Battery cell bypass protection}

Each of the 108 battery cells (54 in each battery) has electro-mechanical bypass protection to prevent an individual cell failing "open" causing the loss of all 54 cells within a battery. If an open cell occurs, the battery will continue to operate minus the one failed cell. This added cell bypass circuitry was implemented after initial design. At the time, some experts considered this addition unnecessary as a cell failing open was an unlikely scenario.

\subsection{Capillary Pumped Heat Transport System (CPHTS)}

The CPHTS It continues to perform nominally. It performs active temperature control of the ASTER-SWIR, ASTERTIR, and MOPITT instruments with no moving parts. The temperature control is accomplished by the use of heaters. The CPHTS loop is managed by setting the reservoir temperature and configuration of CPHTS heaters. Only one loop per instrument is running, moving the heat generated by the instrument to the coldplate. Additional heaters may be turned on to keep the loop running if necessary. 


\subsection{Tracking Data Relay Satellite On-Board Navigation System (TONS)}

TONS perform orbit determination by utilizing Doppler measurements from the Tracking and Data Relay Satellite (TDRS). These measurements allow TONS to estimate Terra's position, velocity, drag, and master oscillator frequency bias. TONS was initially tested on the Extreme Ultraviolet Explorer (EUVE) mission launched in June 1992 [5].

\section{CHALLENGES}

\subsection{Conjunction Assessment - a New Paradigm}

In the early years of the Terra mission, the Flight Operations Team (FOT) focused on maintaining the spacecraft Health \& Safety, maintaining orbit, performing calibration activities, and ensuring the science data downlink. Since then, conjunction assessment has become a new high priority in order to protect Terra and other satellites, and to preserve popular orbits from additional debris. The conjunction assessment process evaluates not only the conjunction of two objects, but also the post-maneuver conjunctions to provide insight on the safety of a planned maneuver. Terra became the first satellite in the $705 \mathrm{~km}$ International Constellation to perform a Risk Mitigation Maneuver (RMM) on October 21, 2005.

This evolving threat was exacerbated by the Chinese anti-satellite missile test with Fengyun 1C on January 11, 2007 and the Iridium 33/COSMOS 2251 collision on February 10, 2009. As of July 2014, Terra has conducted 12 RMMs which included either (a) performing a maneuver to mitigate a conjunction or (b) waiving off a planned maneuver to prevent a post maneuver conjunction. Another 12 conjunctions required RMM planning but were waived off because the risk of a conjunction was reduced to an acceptable level prior to maneuver execution.

\subsection{Micro Meteor Orbital Debris (MMOD)}

Micro Meteor Orbital Debris (MMOD) is a concern. It is believed to be the most likely cause of the BBAT cell failure (1 of 54) and the loss of heater control for half of the BBAT (PBAT remains unaffected).

To compensate for the lost heater control, BBAT has been configured to perform slight overcharge to maintain cell temperatures above freezing (Fig. 5). Recently, the FOT used the BPC to shift additional load to the BBAT and less load to the PBAT. This increased BBAT discharge every orbit has increased the BBAT cold cells by approximately 2 degrees Celsius thereby reducing the risk of BBAT cell reaching freezing temperatures if a load shedding event were to occur without the FOT being able to take action within a few orbits. The FOT accomplished this by using the BPC design in a way that was not intended. After consultation with the spacecraft manufacturer, Goddard Space Flight Center (GSFC) battery experts and Flight Software Sustaining Engineering, changes were made to the battery management software to allow for the batteries to be operated independently. Disabling the PBAT BPC channel increased the BBAT discharge by $14 \%$.

\subsection{Lack of Bit Synch in Fill Data Blocks}

For an unknown reason, bit synch was not added when Terra fill data blocks were originally designed. This requires Terra to transmit live science data on each and every support in order for the White Sands ground station to lock onto the data (since live science data blocks contain bit synch). However, the ground station does not lock onto the data stream immediately, so this initial data must be replayed later. This has increased the complexity of capturing the data, leading to operator errors/data losses and added complexity in automating of SSR operations. 


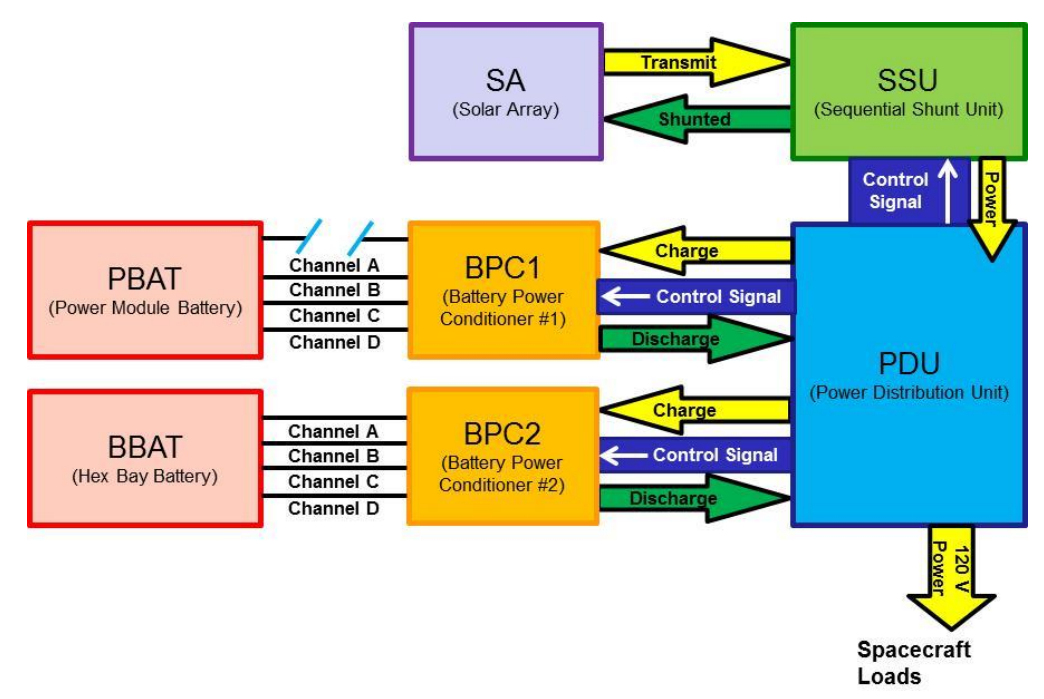

Figure 5. Terra Regulated 120 V Bus Block Diagram with BPC Channel Disabled

\subsection{High Gain Antenna MDA2BITE Anomaly}

The Motor Drive Assembly (MDA) Built-In Test Equipment (BITE) is designed to protect the MDA. If the motor current is too high (above $1250 \mathrm{~mA}$ ) or too low (less than $475 \mathrm{~mA}$ ), the Antenna Control (ACON) automatically turns off the MDA and sets a bit to indicate an MDABITE failure. However, the High Gain Antenna (HGA) MDA opto-couplers are susceptible to single event upsets, induced by high energy protons usually over the South Atlantic Anomaly or the poles, which cause the protection to trip erroneously. When the MDA is turned off, the HGA loses proper pointing control and will begin off-pointing from the intended TDRS. This can result in a drop out of the K-Band (High Rate Science) and SBand (Command/Telemetry) communications links through the HGA until the MDA can be recovered.

This anomaly continues to reoccur, with 2153 recorded MDA2 BITE failures through June 2014 (approximately 12-14 times a month). Due to this issue, the FOT had to develop a Telemetry Monitor (TMON) command to allow the flight software on board to detect the MDA2BITE failure and reset the MDA, recovering within a minute on the first occurrence, minimizing and even eliminating the impact to K-Band and S-Band. After verification of a single event upset, the FOT clears the anomaly flags and returns to normal operations. If a second MDA2BITE failure occurs without the FOT executing the anomaly clean up, the TMON will recover the MDA after a 7 minute wait, and after a 30 minute wait for a third MDA2BITE failure. This is to prevent constantly commanding an MDA recovery in case high frequency MDA2BITE failures are seen due to high radiation or an actual MDA failure. The FOT also has a ground procedure to manually recycle the MDA should the onboard flight software fail to trip.

\subsection{Information Technology Security Threats}

EMOS sustained a significant information technology (IT) security intrusion in Spring 2006 that had the potential to disrupt commanding of the 3 EOS missions. Many ground system security enhancements have since been implemented which greatly improved the EOS ground system security posture and system reliability. These include new hardware, software, and operating systems. The improvements will be vendor-maintained through 2015 , reduce security vulnerabilities, and provide a backup control center.

\subsection{System Obsolescence}

Maintaining aging ground system hardware and deploying required security updates is challenging because of the lack of vendor support for the old hardware and operating systems. Testing and transitioning to new systems with minimal impact to operations requires extensive coordination and replanning around spacecraft anomalies and critical activities. Also, ever-increasing federal and NASA security requirements are adding to the system administration and licensing costs; these are likely to increase in order to stay current with the latest security policies and measures. 


\section{MISSION OPERATIONS}

\subsection{Mission Operations Management}

The Earth Science Mission Operations (ESMO) Project at GSFC is responsible for and conducts mission operations 24 hours a day, 7 days a week. "Mission operations" includes the personnel, systems (hardware and software) and facilities that perform flight operations, the data capture and level zero processing of the science and telemetry data, flight dynamics support, flight software maintenance, and communications. It also covers sustaining engineering and maintenance functions. ESMO coordinates with the members of the Morning Constellation of earth observing satellites (i.e., Landsat7, Landsat-8, Terra and EO-1) to ensure safe operations and conformance to the agreed-upon orbital configuration.

\subsection{Flight Operations}

Daily activities at the EOS Operations Center (EOC) at GSFC include planning and scheduling, command and control, and health and safety monitoring of the spacecraft and instruments. The instrument operations teams (IOTs) at the U.S. and International Partner facilities interface with the EOC daily, submitting plans and schedules, which are used to create an integrated spacecraft and instrument command load that is sent to the spacecraft daily. The FOT monitors Terra spacecraft/instrument health and safety every orbit (14 orbits a day), analyzes spacecraft data, and maintains a log of daily operations.

The FOT notifies the IOTs and management whenever anomalies occur, and consults appropriate experts to resolve anomalies. The FOT has successfully planned, managed, and executed thousands of on-orbit activities over the Terra Mission lifetime. The team conducts approximately 11,000 contacts per year with the Space Network and the Ground Network. In addition to normal daily activities of loading stored commands and navigation tables, the FOT manages the on-board SSR every orbit.

\subsection{Data Capture and Level Zero Processing Operations}

Due to limited SSR capacity, Terra data are played back twice every orbit (99 minutes) via the Tracking and Data Relay Satellite System (TDRSS) to the White Sands ground station in New Mexico. Terra downlinks data via X-band to the polar ground stations in Norway and Alaska whenever TDRSS contacts are not available. Raw data are captured by the EOS Data and Operations System (EDOS) units at the site and forwarded via high speed communications links to EDOS' level zero processing facility at GSFC. Level zero data sets are sent to Distributed Active Archive Centers (DAACs) in the U.S. and the ASTER ground system in Japan via high speed U.S. and Pacific communications networks. Over the life of the mission, the team has recovered $99.3 \%$ of the science data.

\subsection{Ground Systems}

The EOS Mission Operations Ground System (Fig. 6) consists of the EOS Mission Operations System (EMOS), the EDOS, Networks, the EOS Flight Dynamics System (FDS) and the Constellation Coordination System (CCS).

\subsubsection{EOS Mission Operations System (EMOS)}

The EOS Mission Operations Ground System consists of the EOS Mission Operations System (EMOS). EMOS is the control center system resident at the EOC. The design, network architecture, and majority of the current operational hardware for EMOS date back to 1997 1998. The robust fault tolerant architecture has provided reliable with near zero down time for Terra, Aqua, and Aura.

EMOS requires network connectivity to EDOS, the DAACs, TDRSS/White Sands, the GN, the Wallops Flight Facility, and the remote U.S. and International Partner instrument sites. Some software/hardware refresh and enhancements were included when Aqua and Aura operations systems were implemented (2000 to 2003) but funding was not available to upgrade the overall system architecture or hardware. 


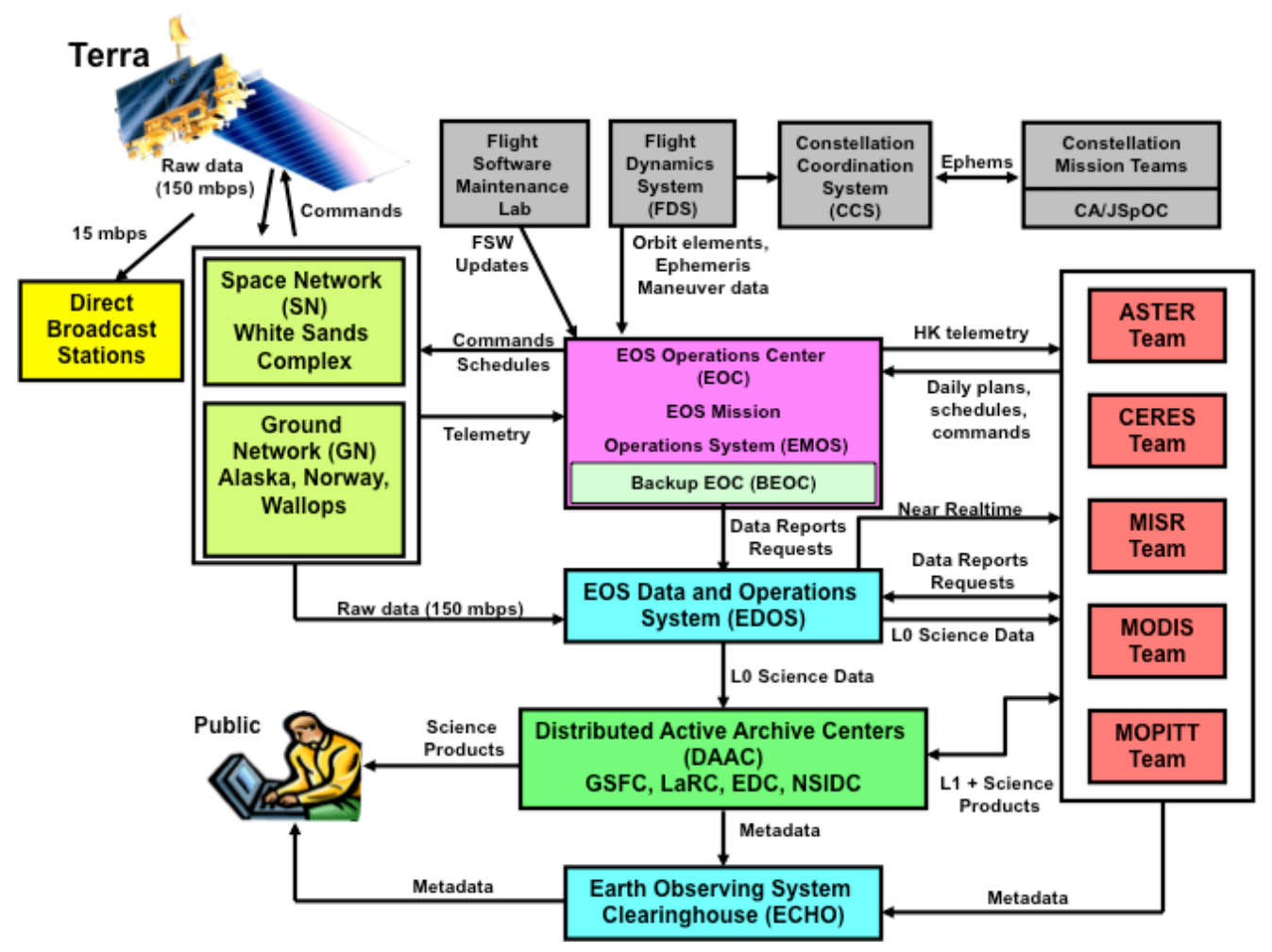

Figure 6. Terra Ground System

\subsubsection{EOS Data and Operations System (EDOS)}

The EDOS has been performing $24 \times 7$ data capture and level zero processing operations since the launch of Terra in 1999 . EDOS has been upgraded through the years to accommodate each new EOS mission (Terra, Aqua, Aura, ICESat, EO-1, and most recently, the Orbiting Carbon Observatory-2 [OCO-2]). EDOS high-rate capture systems were installed to handle X-band downlinks from EOS spacecraft at the ground stations in Alaska (Gilmore Creek), the Svalbard Ground Station in Norway, and the Wallops Flight Facility, in addition to White Sands. EDOS has successfully met and exceeded its requirements to capture, process and deliver level-zero products to end users worldwide.

EDOS is actively pursuing new missions to augment the existing multi-mission set. By leveraging existing EDOS infrastructure/resources across more missions, EDOS can provide substantial savings across all supported Earth Science missions.

\subsubsection{EOS Flight Dynamics System (FDS) and Constellation Coordination System (CCS)}

The EOS FDS and the CCS provide daily flight dynamics products and analysis support necessary for the Terra, Aqua, and Aura mission operations. The products include planning products used by the FOT and Science Teams, orbit maintenance maneuver planning and post-maneuver calibration capabilities, and realtime attitude monitoring and attitude performance validation functions. The FDS is comprised of a suite of computers, servers, and data storage facilities located in the EOC.

The CCS is a software/hardware system designed to facilitate the exchange of critical coordination information between member missions of the Morning and Afternoon Constellations. CCS monitors the orbital configuration of the 2 constellations and provides warnings of predicted close approaches.

\section{FUTURE PLANS}

The Terra team is currently evaluating options to maximize science operations.

Option 1: Terra may continue performing maneuvers to maintain $705 \mathrm{~km}$ frozen orbit, but allow the orbit's MLT to drift 
from 10:30 a.m. to 10:15 a.m. (Fig. 7). When the MLT reaches 10:15 a.m. (the minimum mission requirement) in August 2019, Terra would then safely exit the International Constellation orbit in accordance with constellation guidelines. The orbit lowering will cause the MLT to turn around and start to increase. Terra can continue to collect science data at the lower orbit until the minimum MLT mission requirement is reached again. Based on the remaining fuel, it is estimated that the Terra satellite would be passivated and the mission ended in April 2022.

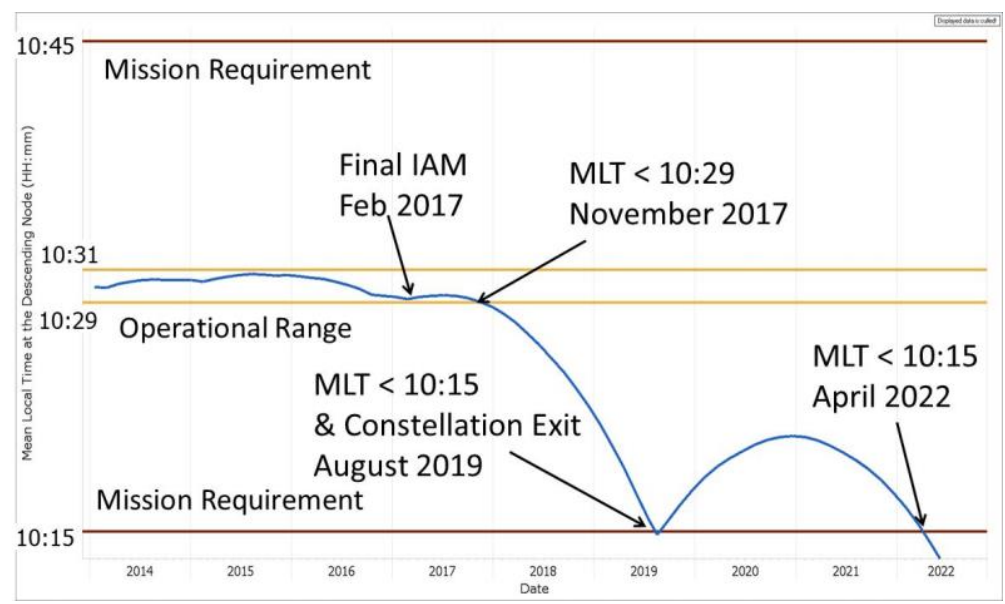

Figure 7. Terra Operations after Constellation Exit

Option 2: Terra would be allowed to operations within its MLT requirements at $705 \mathrm{~km}$ until November 2022; however, it will no longer meet the current constellation exit guidelines. This option has to be evaluated for safety with respect to other constellation members and would require approval by both the International Constellation Team Members as well as NASA Headquarters. Figures 8 and 9 show the predicted Fuel Usage and MLT trends with this option, respectively.

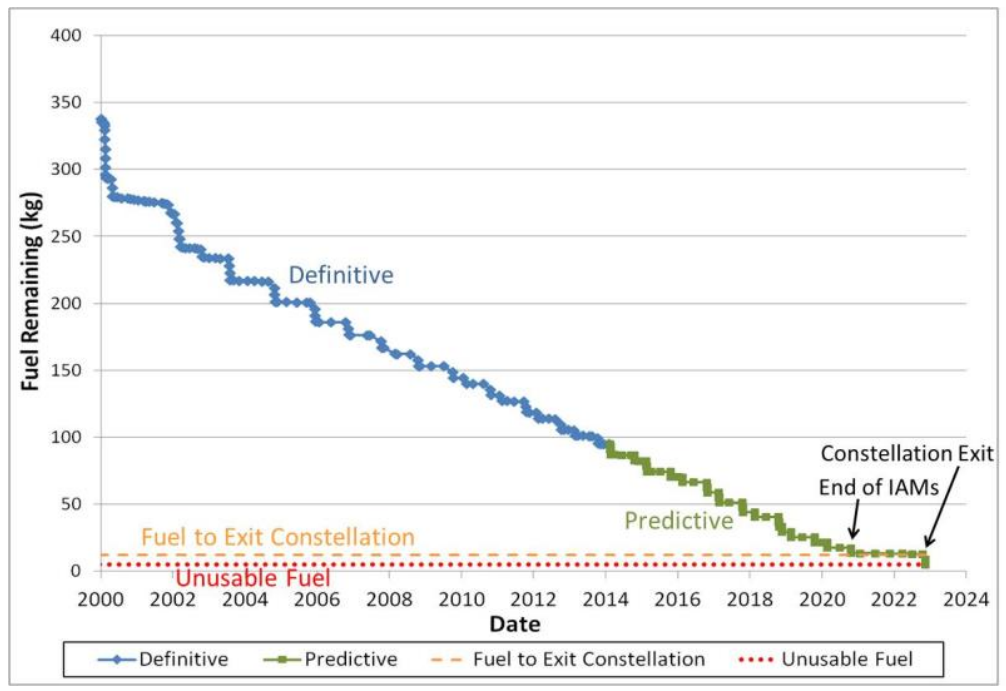

Figure 8. Terra Fuel Usage under Option 2 (Actual and Predicted) for 2000 - 2022 


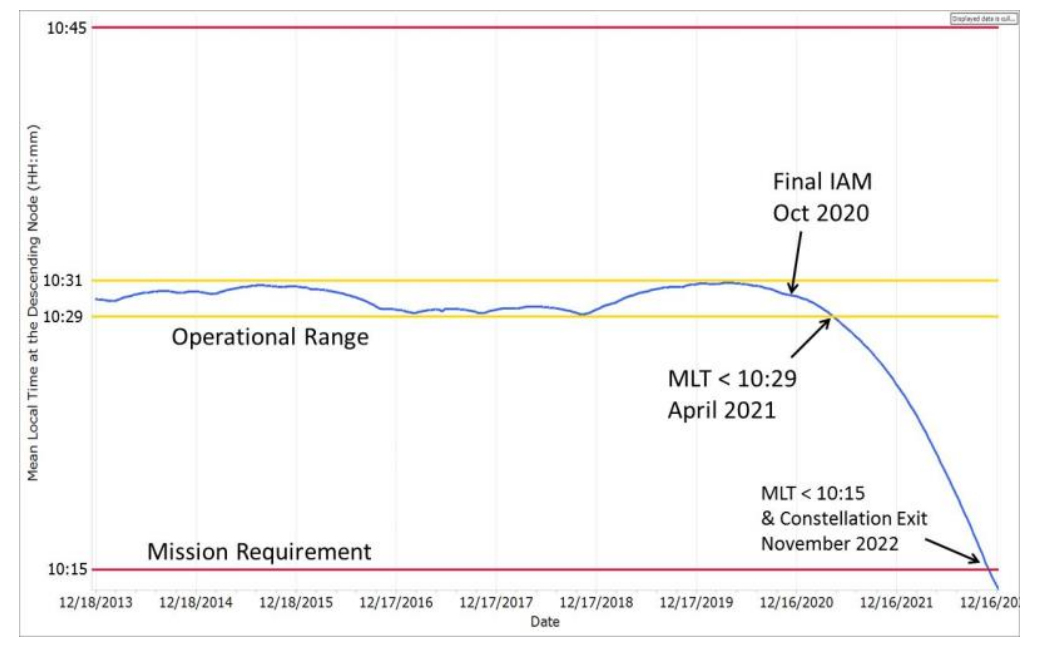

Figure 9. Terra Mean Local Time Evolution under Option 2 (Actual and Predicted) for 2013 - 2022

\section{SUMMARY}

Fifteen years of outstanding science from a satellite that was originally designed for 5 years with a goal of 6 years is definitely worth celebrating. Barring an unexpected contingency, there is enough fuel to continue operations for another 8 years. Terra spacecraft bus is still operating on prime hardware. The instruments are continuing science observations and the scientists are generating new data products. There is no replacement Terra mission on the horizon, so the instrument and mission operations teams are committed to keeping Terra operating as long as possible, adding to the 15-year data record for understanding the Earth and benefiting everyday needs in the areas of fire monitoring and management, fishery, disaster recovery and management, and other applications.

\section{ACKNOWLEDGEMENTS}

The future looks promising for more years of Terra science data thanks to the great cooperation between the Terra teams, the excellent workmanship in the Terra spacecraft and instruments, and the support of NASA Headquarters, the worldwide Earth science community and our International Partners.

\section{REFERENCES}

[1] NASA," Terra Mission (EOS/AM-1)”, EO Sharing Earth Observation Resources, https://directory.eoportal.org/web/eoportal/satellite-missions/t/terra[2] Turner, G. F. and Hill, H. C., "STS 41-D SOLAR ARRAY FLIGHT EXPERIMENT", http://commons.erau.edu/cgi/viewcontent.cgi?article=2408\&context=spacecongress-proceedings, Space Congress Proceedings

[3] NASA, "STS-41D”, NASA web site, 1984, http://www.nasa.gov/mission_pages/shuttle/shuttlemissions/archives/sts$\underline{41 \mathrm{D} . h t m l}$

[4] Keys, D., "Earth Observing System (EOS) Terra Spacecraft 120 Volt Power Subsystem: Requirements, Development and Implementation", NRTS, 2000, http://ntrs.nasa.gov/archive/nasa/casi.ntrs.nasa.gov/20000070467.pdf

[5] Gramling, C. J., Hart, R. C., Teles, J., Long, A. C., and Maher, M. J., "Performance assessment of the TDRSS Onboard Navigation System (TONS) experiment on EP/EUVE," NASA Technical Reports Server (NTRS), 1 January 1993, http://ntrs.nasa.gov/search.jsp?R=19950054124 